\title{
The impact of accelerator processors for high-throughput molecular modeling and simulation
}

\author{
G. Giupponi \\ Computational Biochemistry and Biophysics Lab, GRID IMIM Universitat \\ Pompeu Fabra, Barcelona Biomedical Research Park (PRBB), C/ Doctor \\ Aiguader 88, 08003 Barcelona, Spain
}

M. J. Harvey

Information and Communications Technologies, Imperial College London, South Kensington, London, $S W^{7}$ 2AZ, UK

\section{G. De Fabritiis}

Computational Biochemistry and Biophysics Lab, GRIB IMIM Universitat

Pompeu Fabra, Barcelona Biomedical Research Park (PRBB), C/ Doctor

Aiguader 88, 08003 Barcelona, Spain

\begin{abstract}
The recent introduction of cost-effective accelerator processors (APs), such as the IBM Cell processor and Nvidia's graphics processing units (GPUs), represents an important technological innovation which promises to unleash the full potential of atomistic molecular modeling and simulation for the biotechnology industry. Present accelerator processors can deliver over an order of magnitude more floatingpoint operations per second (flops) than standard processors, broadly equivalent to a decade of Moore's law growth, and significantly reduce the cost of current atombased molecular simulations. In conjunction with distributed and grid computing solutions, accelerated molecular simulations may finally be used to extend current in silico protocols by use of accurate thermodynamic calculations instead of approximate methods and simulate hundreds of protein-ligand complexes with full molecular specificity, a crucial requirement of in silico drug discovery workflows.

Teaser phrase: New accelerated computing devices will unleash the predictive power of molecular modeling and simulation for biotechnology.
\end{abstract}

Key words: Cell processor, graphics processing units (GPUs), accelerated computing, molecular modeling and simulation, drug discovery, distributed and grid computing 
Bringing a new drug to market is a long and expensive process[1] encompassing theoretical modeling, chemical synthesis and experimental and clinical trials. Despite the considerable growth of biotechnologies in the last ten years, the practical consequences of these techniques on the number of approved drugs has failed to meet expectation[2]. Nonetheless, the discovery process greatly benefits from the use of computational modeling[3], at least in the initial stages of compound discovery, screening and optimization[4].

Among the techniques available, force-based molecular modeling methods (briefly, molecular modeling) such as molecular dynamics are particularly useful in studying molecular processes at the atomistic level, providing accurate information on macromolecular dynamics and thermodynamic properties. Bridging from the molecular-atomistic (femtosecond) to biological (micromillisecond) timescales is still an unaccomplished feat in computational biology: the complexity of the modeling impedes sufficient sampling of the evolution of the system, even on expensive high performance computing (HPC) resources. For instance, cost and sampling constraints have so far limited a routine molecular dynamics run over a PC cluster to a single protein system for tens of nanoseconds, barely sufficient to compute the binding free energy using an exact thermodynamic method [5]. This information is of great importance in the discovery process for virtual screening, lead optimization and in silico drug discovery [4], but needs to be computed for hundreds of protein-ligand systems efficiently and economically in order to open the way for molecular simulations to become a routine tool in the discovery workflows used in the biotechnology industry.

In light of the significant changes that have occurred lately in microprocessor design, we review the first cost-effective accelerator processors (APs) - the Cell processor and Nvidia graphics processing units (GPUs) - and examine results of their early adoption in stand-alone and grid-computing scenarios in the context of high-throughput molecular simulation for biomolecular applications.

\section{Accelerator processors}

Historically, microprocessor performance has improved primarily through the raising of clock speeds, made possible by the development of ever-finer fabri-

Email addresses: giovanni.giupponi@upf .edu (G. Giupponi), m.j.harvey@imperial.ac.uk (M. J. Harvey), gianni.defabritiis@upf.edu (G. De Fabritiis). 


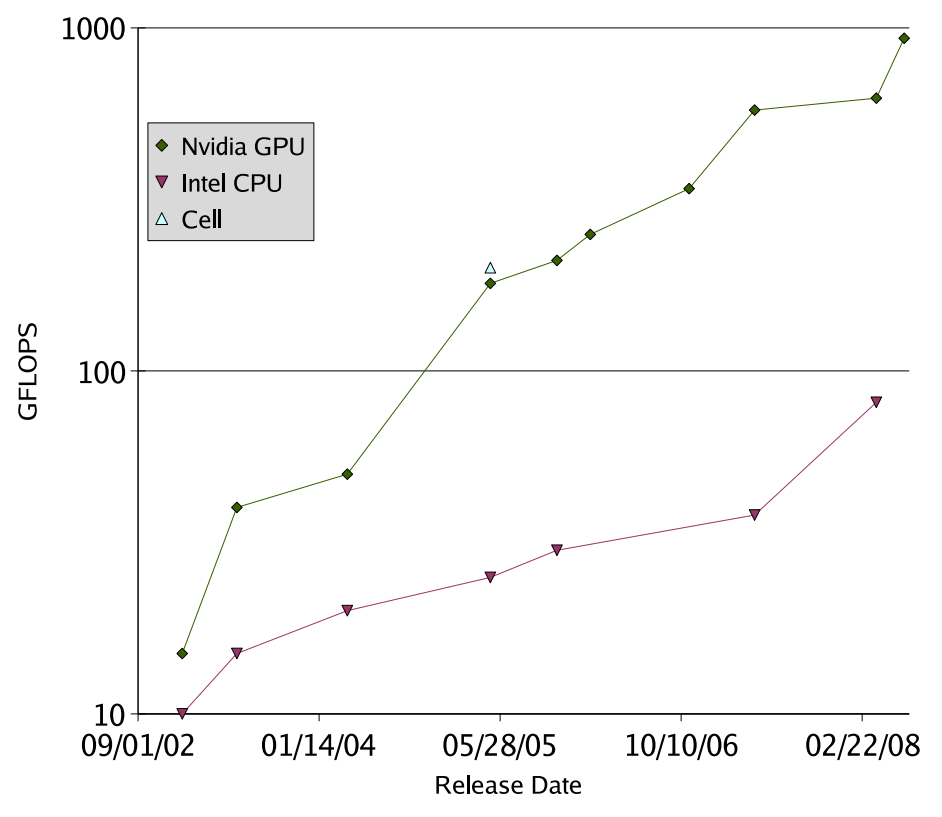

Fig. 1. The theoretical peak performance trend of Nvidia and Cell processors in comparison to contemporary Intel processors. Gflops measures for Nvidia and Intel devices are taken from [11]. Since 2002, Nvidia GPU performance has increased by $\approx 100 \%$ /annum whilst that of Intel CPUs by $\approx 50 \%$ /annum. Nvidia GPU devices gained the programmable flexibility necessary for general purpose computation with the release of the G80 core in November 2006.

cation processes. In recent years, it has become increasingly difficult to keep increasing clock speeds because of fundamental limits in the process technology and power consumption. Performance has also been limited by the increasing relative cost of accessing main memory, the speed of which has increased at a slower rate than CPUs. Despite this, Moore's Law[6] (Figure 1), the empirical observation that the density of transistors on an integrated circuit doubles every $18-24$ months has continued to hold true. Manufacturers have been forced to reconsider their 'single fast core' design and have used the greater transistor counts to build CPUs containing multiple independent processing cores. Even so, a large fraction of the transistors on a modern CPU remain devoted to providing a very fast cache memory that is used to hide the cost of communicating to the much larger but slower main system memory.

As a result, although the aggregate performance of multi-core CPUs has continued to increase, it is no longer possible for serial (single-threaded) programs to take advantage of the increased processing capability, as the computing cores are independent. Instead, it is necessary for codes to be parallelised: adapted to perform computation concurrently on multiple cores. In the case of molecular dynamics (MD) modeling, parallel codes such as NAMD [7] may be used to distribute simulations across multiple processors. Low latency, high bandwidth interconnections between processors are needed for good scalability and performance is ultimately limited by the size of the simulated system 

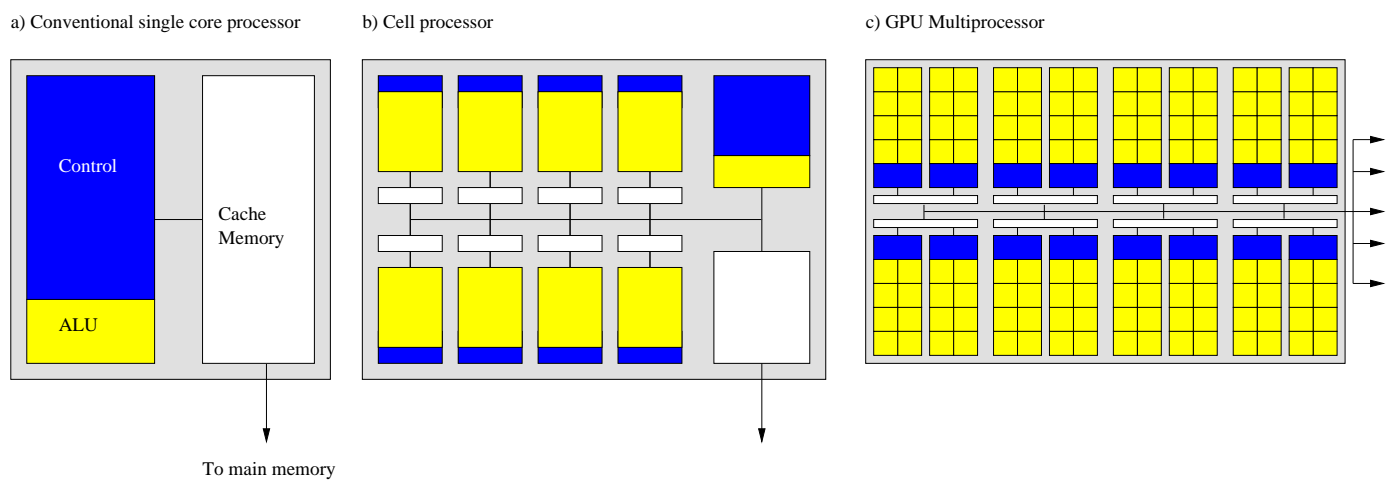

Fig. 2. Illustration of the relative differences between CPU, Cell and GPU designs. (a) A conventional CPU dedicates a relatively large fraction of its transistors to complex control logic, to maximise performance on a mixed workload of serial code. A large cache memory is required to disguise the cost of accessing slow main memory.

(b) The Cell processor contains 8 Synergetic Processing Elements (SPEs) designed to maximise arithmetic throughput at the expense of the ability to run complex programs. A conventional CPU core is used to control the SPEs and supply them with data. (c) Nvidia GPU devices have a very large number of cores, all of which may talk to main memory directly. There is no cache for main memory accesses, the cost of which is hidden by overlapping many computational threads. The overall program execution is controlled by code running on the CPU of the host system.

which must increase with processor count in order to maintain parallel efficiency.

An alternative approach is to employ special purpose hardware specifically tailored for molecular dynamics simulation, such as MD-GRAPE [8] or Anton [9]. These can yield an improvement in performance of several orders of magnitude over commodity processors, however special hardware is expensive to buy, develop and - without continued development - Moore's Law ensures that gains in performance relative to general purpose computing hardware are rapidly eroded.

Recently, hardware manufacturers have introduced a third class of devices, which we term accelerator processors (AP). These vary in architectural details but share the common trend of having very many, comparatively simple processing cores in a single package. These cores are generally optimised to have much higher floating-point arithmetic performance than conventional CPUs (Figure 1), at the expense of being able to execute complex branching programs efficiently, for example reduced control hardware (see Figure 2). At present, each vendor is independently developing its solutions, with SonyToshiba-IBM (STI) Cell processor (Figure 2b) and Nvidia graphics processing units (GPUs) (Figure 2c) already adopted by a large user-base. Cell and GPU devices are able to achieve an order of magnitude more floating point operations per second (flops) than conventional processors at a similar price and, for some numerically intensive applications, promise a speed-up of almost 100 
times. Furthermore, because these devices are designed for the mass market, they are also substantially cheaper than previous special purpose hardware.

The main specifications of currently available devices are as follows:

- The Cell processor architecture

Sony-Toshiba-IBM released in 2006 the first general-purpose processor (Cell) [10] that implements a multi-core architecture (9 inhomogeneous cores) with features specifically designed to mitigate the effects of memory access latency. At more than 230 Gflops (230 billion flops) in single precision floatingpoint, the Cell provides 10 times more flops than a CPU at similar cost (see Box 1).

- The GPU architecture

The introduction of general-purpose programmable capabilities to graphics processing units has marked a drastic change in hardware and high performance computing architectures, as graphic cards can now be used for the computing intensive parts of a calculation. The Nvidia G80 architecture, introduced in 2007, is designed to be well-suited to data-parallel computation, in which the same program is executed on many data elements in parallel [11]. Current products based on this architecture are able to achieve up to 648 Gflops (GeForce 9800GTX) (see Box 2).

By comparison, a dual core Intel Xeon 5160 is capable of approximately 38 Gflops whilst Intel's fastest quad-core processor, the Xeon 5472 achieves 81 Gflops, but at a considerable cost premium[12]. Other major manufacturers such as AMD-ATI and Intel have announced their intention to enter the accelerator processor market, but a systematic evaluation of architecture and performance for their products is at the moment not possible.

\section{Costs, benefits and risks}

\section{Software re-engineering}

Although APs offer high peak computational performance, exploiting this efficiently comes at the cost of ease of use: codes must be re-factored as highly parallel programs. Code redesign and redevelopment has a very high cost that, when weighted against the traditional "free" performance increase provided by the next iteration of conventional hardware, has in the past significantly limited the appeal of special-purpose hardware on the high-performance computing market. In the next few years, this re-engineering cost will become less important as all serial code will have to be redesigned to take advantage of standard multi-core processors. Therefore, the possibility of realising a 100 times improvement in application performance on an AP, broadly equivalent 
to a decade of Moore's Law growth, makes code redevelopment a fully justified proposition to speed-up current workflows or perform totally new applications that could not be achieved on single CPUs within the next decade.

With wide and pervasive availability of APs across the market, from highend high performance computing [13] to commodity Nvidia graphics cards, we contend that applications programmers must eventually adopt this technology, with the risk of being seriously outperformed otherwise, however embracing accelerator processors requires the acquisition of new know-how and a commitment to develop further application enhancements on such architectures. Porting an application to either the Cell or Nvidia GPUs requires re-factoring code to conform the platform's programming model. The Cell processor can be programmed as a multi-core chip using standard ANSI C and relying on libraries from the IBM system development kit (SDK)[14] to handle communication, synchronization and Single Instruction Multiple Data (SIMD) computation. The SIMD model is similar to that found on some commodity CPUs, such as Altivec (PowerPC) and SSE (Intel). The Nvidia's Complete Unified Device Architecture (CUDA) SDK[11] reduces the difficulty of programming GPU devices by providing a minimal set of extensions to the standard $\mathrm{C}$ programming language. Code functions written to be executed on the GPU are known as kernels and are executed in blocks. Each block consists of multiple instances of the kernel, called threads, that are run concurrently on a single multiprocessor. The CUDA run-time is responsible for efficiently scheduling the execution of blocks on available GPU hardware. As the CUDA programming model abstracts the implementation details of the GPU, the programmer may easily write code that is portable between current and future Nvidia GPUs. Furthermore, it is expected that future CUDA SDKs will provide support for targeting Intel and AMD multi-core CPUs, although obviously without the performance boost provided by GPU hardware.

\section{Hardware costs and energy savings}

Accelerator processors are already available as consumer products: the Sony PlayStation 3 (PS3) features a Cell processor whilst Nvidia's entire range of GeForce graphics cards support CUDA at varying levels of performance. Professional-grade variants of these products are also available in the form of IBM Cell QS20 blade servers and Nvidia's Tesla GPU range. Mass production assures low unit price, better vendor support and a broad market. Millions of PS3s have been sold and can be trivially converted to running workstation operating systems such as Linux[15]. GPUs can be bought and added to most PCs as a simple end-user upgrade. APs cost a few hundred US dollars per piece and approach the computational power of small (tens of machines) PC clusters. Additionally, adopting APs may markedly reduce setup, support and running costs in comparison to a conventional cluster. It has been noted that the PS3 
is one of the most efficient hardware architectures per dollar for molecular dynamics[16] and fluid dynamics.

APs are also very energy efficient computing resources. For example, it is claimed that the recently announced ATI Firestream GPU board will achieve 5 Gflops/Watt [17], a figure to be matched by Nvidia's next generation of Tesla products [18]. As for the Cell processor, a PS3 has a peak power rating of 280W[19], delivering at least 0.8 Gflops/Watt, and more professional solutions such as IBM QS21 blades featuring 2 Cell processors deliver 2.09[20] Gflops/Watt. Furthermore, IBM has recently fabricated the Cell processor on a 45 nanometre process, estimating a $40 \%$ less power consumption. As of July 2008, the most energy efficient supercomputer delivers 0.35 Gflops/Watt[21], therefore standard computer clusters are at least one order of magnitude less efficient than APs. These figures sum up the significant impact that APs will have not only performance, but also on green computing.

At the lowest level of their accelerated-computing infrastructure, a company could use a locally deployed BOINC installation - similar to that used by gpugrid.net - to run simulations at zero cost using GPU hardware already available in office desktop computers. A scenario in which the simulations are mission critical might involve the use of Tesla units (approximately 5,000 US dollars per blade with 4 teraflops per blade) or properly engineered GeForce clusters (approximately 2,000 US dollars for 4 teraflops). Obtaining the same computing power on a cluster of CPUs would cost over an order of magnitude more in hardware and substantially more to run in terms of power and maintenance.

\section{Accelerated modeling}

Despite the very recent introduction of APs into the market, a variety of applications targeting different industrial and scientific fields have already appeared. Excellent performance speed-ups have been reported for such diverse cases as computational finance[22], fluid dynamics[23], sequence alignment[24] and quantum chemistry[25]. These notable achievements for such a variety of algorithms highlight the potential of APs for computational science in general.

Accelerators processors will prove to be beneficial where the application has potential for a large degree of parallelism. In this sense, they should be appropriate for most of the computational biology tools used in the industry (network building, cell and organ simulations) and for multidimensional modeling of properties like ADME/Tox alongside bioactivity [26]. These tools are yet to be ported on accelerator processors, a task which may require significant re-engineering. We would expect that docking programs will be one of the first 
applications which will be available on the new hardware, with molecular dynamics simulations currently being the most active sector of development. For quantum chemistry codes, which are computationally very expensive, accelerator processors could play a major role. A current limitation for this kind of application is the lack of double precision support of current GPUs. With new cards coming out late in the year featuring double precision, it is likely that quantum mechanics code developers will be looking at this hardware technology. Software vendors will require some time to perform this migration, but the different exponential growth of GPU vs CPU shown in Figure 1, will ultimately force this process.

We review here in more detail results that are directly relevant to extending current molecular modeling capabilities. Several groups have started to implemented MD routines on APs. Meel et al[27] describe a CUDA implementation of Lennard-Jones MD which achieves a net speedup of up to 40 times over a conventional CPU. Although suitable for coarse-grained simulations, the lack of support for an atomistic, biomolecular force field limits the applicability of the code. Stone et al [28] demonstrate the GPU-accelerated computation of the electrostatic and van der Waals forces obtaining $10-100$ times speed-up compared to heavily optimised CPU-based implementations, but because the remainder of the MD simulation remains performed by the host CPU, the net speed-up is reduced to around 5 times. Preliminary results for a fully atomistic MD program called aceMD[29] show a 50 times speed-up measured at the peak performance of a parallel multi-GPU MD code. Stone et al also describe performance improvement for other algorithms in the visualization software VMD [30] such as Coulomb-based ion placement and time-averaged potentials calculations obtaining respectively up to 470 and 110 performance speed-up. On the Cell processor, a sustained performance of 30 Gflops was achieved for a fully atomistic molecular dynamics program, more than an order of magnitude faster compared to the same application running on a single processor core[16]. We note here that all performance speed-ups in these studies have been obtained using single precision floating-point, which is adequate for MD simulations. Nevertheless, it is expected that double precision arithmetic will be supported in the next iterations of the Cell processor and GPUs.

\section{Distributed computing in the accelerated era}

Computational grids enable scientists to distribute simulations across a pool of machines in order to benefit from their aggregate power, and have already proved useful for fast calculations of binding affinities using MD techniques[31]. Due to the computational cost of molecular simulations, the grid is usually composed of very expensive parallel-processing HPC resources, the costs of which limit the applicability of the approach. When distributed computing is 
combined with AP-equipped hardware, however, a single computational node is sufficiently powerful (equivalent to tens of CPUs) to simulate molecular dynamics trajectories of reasonable length in a day for a molecular system of the order of 50,000 atoms. This speedup, compared to a couple of weeks for the same run on a single PC, enables the effective use of loosely-coupled computational grids of AP-equipped machines for molecular dynamics simulations.

Inevitably, making efficient use of a collection of non-dedicated machines presents new challenges. As the machines might not be dedicated to computing during office hours, the pool of machines available must be treated as transient: the owner of the GPU-equipped PC may choose to power it down at any moment, for example. The distributed infrastructure must accommodate this and be able to correct for the loss of results arising from an incomplete simulation. Software like the Berkeley Open Infrastructure for Network Computing (BOINC) framework[32] or Condor [33] distributed computing solutions have reached, over many years, the maturity and stability required by these type of applications (Seti@HOME [34]). For instance in the ps3grid.net and gpugrid.net projects [35] a BOINC server produces hundreds of calculations a day with very little human intervention and very high reliability on a diverse range of hardware including PlayStation3 and PC-based Nvidia GPUs scattered across the globe. Similarly, a dedicated in-house distributed grid of Nvidia graphics cards, commonly already in use in most modern PC, could reach throughput only possible with use of the largest HPC resources, but at fraction of the cost.

A simple benchmark of the impact of distributed computing using accelerator processors is given by the first numerical experiment of ps3grid.net which used steered molecular dynamics to compute the free energy of translocation of a potassium ion across a transmembrane pore[36]. ps3grid.net uses an MD code that is optimised for the Cell processor[16] and runs a single MD simulation per client. The trajectories produced by this ensemble of simulations are subjected to statistical analysis[36] to recover the free energy profile of the ion translocation. The setup of the computational protocol was first performed using standard supercomputing hardware with a few iterations of over 50 runs, each lasting half day on 32 processors and amounting to 19,000 CPU hours and around $40 \mathrm{~ns}$ of simulation time [36]. An extended set of numerical experiments run on ps3grid.net produced 5, 000 trajectories, 4 microseconds of simulated time, over 200 years of CPU time with a daily output of $100 \mathrm{~ns}$ and 5 GB of data. This numerical experiment produced a number of pullings which is at least one order of magnitude closer to single-molecule pulling experiments performed using optical tweezers[37]. 


\section{Future outlook for medium-throughput molecular modeling}

There is great interest in methods for supporting and optimising experimental high-throughput screening $[4,38]$ in order to identify, characterise and optimise possible leads for a given target out of the vast number of viable chemical compounds. It is, however, very difficult for such methods to account correctly for the many phenomena involved in complex formation, such as the subtle interplay between entropy and enthalpy, conformational changes of the ligand or the substrate, presence of water molecules in the binding sites and limited resolution of structures[39,40]. We advocate that high-throughput molecularbased methods aimed at a detailed, systematic and physically-sound quantitative description of the binding site can now be used thanks to accelerator processors.

Calculating the chemical potential of water[41] in the binding site already provides a cheap but useful way to understand the mechanism of ligand-protein binding. Thermodynamics integration (TI) [31], steered molecular dynamics [36], umbrella sampling [5] and linear interaction energy (LIE) approaches [42] can be applied to several hundred protein structures at once. Furthermore, standard molecular dynamics could be of great help to the understanding of a plethora of molecular and cellular processes. High throughput docking using new techniques or improved scoring functions would become treatable on distributed accelerated solutions even if such methods require substantially more computing power.

Methods using atomistic force-fields and dynamical molecular simulations can provide enough level of detail to achieve accurate virtual screening performance[39]. In fact, such approaches potentially take into account system specific interactions involved in complex formation. We note that as larger timescales and extended sampling become routinely available the limitation of force-fields will start to appear more evidently. Cases where this limitation is already demonstrated are known as in [36] for a gramicidin A pore, where an error of several $\mathrm{kcal} / \mathrm{mol}$ of the energetic barrier of translocation is consistently found, possibly due to lack of polarization in the water molecules within the pore. Nevertheless, current force-fields have shown also encouraging degrees of accuracy. Increasing sampling capabilities will move the bottleneck from computers back into the modeling of molecular structures and classical force-fields with the possibility of developing revised versions which will accommodates the new timescales.

As an example, it is common for a biotechnology company to screen potential targets using docking ranking methods. The availability of accelerated software for molecular dynamics simulations would allow them to perform more accurate thermodynamic free energy protocols as umbrella sampling which 
are expected to produce much higher enrichment than ranking methods. Of course, as there are several possible compounds to test the problem quickly becomes combinatorially too expensive to treat experimentally. Supplementing the experimental work with free-energy calculations performed across an in-house distributed GPU computing solution using quantitative thermodynamic calculations could significantly reduce the cost and time of the research provided that the thermodynamic screening can be performed on at least hundreds of protein-ligand complexes, rather than just few as it is for current standard CPU hardware solutions. The key factor introduced by accelerators is to shift the modelling from single binding affinity predictions to high-throughput affinity predictions.

\section{Conclusion}

Accelerator processors have the potential to provide a radical change in scientific and industrial computation. With an effective performance tens of times that of standard computers and doubling each 8-12 months, unprecedented levels of computational power can be put into the hands of scientists and programmers at an affordable cost. Such disruptive technology is already being used by research groups and companies in many different fields, and applications targeting molecular modeling are appearing at a steady pace. The gain of raw performance combined with the possibility of distributed, grid-like deployment, can finally unleash the full potential of advanced molecular modeling methods, routinely allowing virtual experiments previously only achievable on supercomputers. In effect, these loosely-coupled distributed computing solutions can be seen as grids of small computer clusters provided that the software is optimised to run on accelerated processors.

For the case of molecular modeling and simulations, such novel accelerator hardware and technologies are bound to play a significant role in next generation in silico drug discovery. APs open up the possibility to increase the molecular detail allowing more refined and computationally expensive methods based on atomistic force fields that cannot presently be considered. APs will therefore allow a fundamentally different approach to modeling and reshape in silico drug discovery protocols, by increasing the detail (up to atomistic force-field based level) and using more accurate thermodynamic methods to compute free energies, and by increasing the throughput (more trajectories, longer, on more proteins and ligands) to an extent outside the capability of traditional processors for the next decade. 
We gratefully acknowledge support from Barcelona supercomputing center (http://www.bsc.es), Acellera Ltd. (http://www.acellera.com), Sony Computer Entertainment Spain (http://www.scee.com) and Nvidia corporation (http://www.nvidia.com). We thank Jordi Mestres and Ferran Sanz for a critical reading of the manuscript. GG acknowledges the Aneurist project (http://www.aneurist.org) for financial support. GDF acknowledges support from the Ramon y Cajal scheme.

Conflicts of interest statement: We notify the journal that the authors are scientific consultants and also share holders of Acellera Ltd, a UK based company selling software solutions for accelerated processors.

\section{References}

[1] M. Dickson, J. Gagnon, Key factors in the rising cost of new drug discovery and development., Nat Rev Drug Discov 3 (5) (2004) 417-29.

[2] P. Nightingale, P. Martin, The myth of the biotech revolution, Trends in Biotechnology 22 (11) (2004) 564-569.

[3] W. L. Jorgensen, The many roles of computation in drug discovery., Science 303 (5665) (2004) 1813-1818.

[4] S. Ekins, J. Mestres, B. Testa, In silico pharmacology for drug discovery: methods for virtual ligand screening and profiling, British Journal of Pharmacology 152 (2007) 9-20.

[5] B. Smit, D. Frenkel, Understanding Molecular Simulation, Academic, New York, 2002.

[6] G. Moore, Lithography and the future of Moore's law, Proceedings of SPIE 2438 (1995) 2.

[7] J. Phillips, R. Braun, W. Wang, J. Gumbart, E. Tajkhorshid, E. Villa, C. Chipot, R. Skeel, L. Kale, K. Schulten, Scalable molecular dynamics with NAMD, J. Comput. Chem 26 (16) (2005) 1781-1802.

[8] R. Susukita, T. Ebisuzaki, B. Elmegreen, H. Furusawa, K. Kato, A. Kawai, Y. Kobayashi, T. Koishi, G. McNiven, T. Narumi, et al., Hardware accelerator for molecular dynamics: MDGRAPE-2, Computer Physics Communications 155 (2) (2003) 115-131.

[9] D. Shaw, M. Deneroff, R. Dror, J. Kuskin, R. Larson, J. Salmon, C. Young, B. Batson, K. Bowers, J. Chao, et al., Anton, a special-purpose machine for molecular dynamics simulation, Proceedings of the 34th annual international conference on Computer architecture (2007) 1-12. 
[10] IBM website on the Cell processor, http://www.research.ibm.com/cell/.

[11] Complete Unified Device Architecture, Nvidia, http://developer.nvidia.com/object/cuda.html.

[12] Intel Xeon Processor 5000 Sequence (accessed 29 April 2008) http://www. intel.com/performance/server/xeon/hpcapp.htm.

[13] IBM Roadrunner supercomputer, http://www . lanl.gov/roadrunner.

[14] IBM Cell Broadband Engine Software Development Kit (SDK), http://www.ibm.com/developerworks/power/cell/.

[15] M. Harvey, G. Giupponi, J. Villa-Freixa, G. De Fabritiis, PS3GRID.NET: Building a distributed supercomputer using the PlayStation 3, Distributed and Grid Computing - Science Made Transparent for Everyone. Principles, Applications and Supporting Communities, 2007.

[16] G. De Fabritiis, Performance of the cell processor for biomolecular simulations, Comp. Phys. Comm. 176 (2007) 660.

[17] HPCwire http://www.hpcwire.com/topic/processors/17910894.html.

[18] HPCwire newsletter http://www.hpcwire.com/hpc/2269095.html.

[19] PlayStation3 Safety and Support Manual, Version 2.0, Sony document 3-275578-31(1). http://www.playstation.com/manual/pdf/PS3-02_03-1.9_1.pdf.

[20] J. Morrison, D. Turek, The new era of supercomputing: Insights from the national labs to wall streetIBM http://www03.ibm.com/industries/financialservices/doc/content/bin/ibm_lanl_at_sifma_tech_2007_web.pdf.

[21] The green 500 list http://www.green500.org/lists/2008/02/green500.php.

[22] J. Easton, I. Meents, O. Stephan, H. Zisgen, S. Kato, Porting financial markets applications to the cell broadband engine architecture, Tech. rep. (2007).

[23] Y. Zaho, Lattice boltzmann based pde solver on the gpu, The Visual Computer 24 (2007) 323-333.

[24] S. Manavski, G. Valle, Cuda compatible gpu cards as efficient hardware accelerators for smith-waterman sequence alignment, BMC Bioinformatics 9 (2008) S10.

[25] I. Ufimtsev, T. Martínez, Quantum Chemistry on Graphical Processing Units. 1. Strategies for Two-Electron Integral Evaluation, J. Chem. Theory Comput 4 (2) (2008) 222-231.

[26] P. Swaan, S. Ekins, Reengineering the pharmaceutical industry by crash-testing molecules, Drug Discovery Today 10 (17) (2005) 1191-1200.

[27] J. A. van Meel, A. Arnold, D. Frenkel, S. F. P. Zwart, R. G. Belleman, Harvesting graphics power for md simulations, Mol. Sim. (2008) 259-266. 
[28] J. t. Stone, Accelerating molecular modeling applications with graphics processors, J. Comp. Chem. 28 (2007) 2618-2640.

[29] AceMD website http://multiscalelab.org/acemd .

[30] W. Humphrey, A. Dalke, K. Schulten, VMD: Visual molecular dynamics, Journal of Molecular Graphics 14 (1) (1996) 33-38.

[31] P. Fowler, S. Geroult, S. Jha, G. Waksman, P. Coveney, Rapid, Accurate, and Precise Calculation of Relative Binding Affinities for the SH2 Domain Using a Computational Grid, J. Chem. Theory Comput 3 (3) (2007) 1193-1202.

[32] Berkeley Open Infrastructure for Network Computing http://www.boinc. berkely.edu.

[33] M. Litzkow, M. Livny, M. Mutka, Condor-a hunter of idle workstations, Distributed Computing Systems, 1988., 8th International Conference on (1988) 104-111.

[34] Search for ExtraTerrestrial Intelligence at Home http://www. setiathome. berkeley.edu.

[35] PS3GRID project http://www.ps3grid.net; http://www.gpugrid.net.

[36] G. De Fabritiis, P. V. Coveney, J. Villá-Freixa, Energetics of k+ permeability through gramicidin a by forward-reverse steered molecular dynamics, in press, Proteins doi:10.1002/prot.22036 (2008).

[37] D. Collin, F. Ritort, C. Jarzynski, S. Smith, I. Tinoco Jr, C. Bustamante, Verification of the Crooks fluctuation theorem and recovery of RNA folding free energies, Nature 437 (7056) (2005) 231.

[38] J. Bajorath, Integration of virtual and high-throughput screening., Nat Rev Drug Discov 1 (11) (2002) 882-894.

[39] H. Gohlke, G. Klebe, Approaches to the Description and Prediction of the Binding Affinity of Small-Molecule Ligands to Macromolecular Receptors, Angewandte Chemie International Edition 41 (15) (2002) 2644-2676.

[40] J. E. Ladbury, Just add water! the effect of water on the specificity of proteinligand binding sites and its potential application to drug design., Chem Biol 3 (12) (1996) 973-980.

[41] G. De Fabritiis, S. Geroult, P. V. Coveney, G. Waksman, Insights from the energetics of water binding at the domain-ligand interface of the src sh2 domain, in press, Proteins doi:10.1002/prot.22027 (2008).

[42] E. Stjernschantz, J. Marelius, C. Medina, M. Jacobsson, N. P. E. Vermeulen, C. Oostenbrink, Are automated molecular dynamics simulations and binding free energy calculations realistic tools in lead optimization? an evaluation of the linear interaction energy (lie) method., J Chem Inf Model 46 (5) (2006) $1972-1983$. 
[43] Nvidia GeForce 8800 GPU Architecture Overview, Nvidia Technical Report TB-02787-001_v01 (2006).

[44] Nvidia, http://nvidia.com. 


\section{The Cell processor}

The present version of the Cell processor comprises one general purpose PowerPC processing element (PPE) which runs the operating system and acts as a standard processor and 8 independent, specialised, synergistic processing elements (SPEs) (Figure 2) that are simple vector processors. The essential characteristics are:

- Main memory can be accessed only by the PPE core: each SPE must use its limited in-chip local memory (local store) of $256 \mathrm{~KB}$. This memory is accessed directly without any intermediate caching.

- Optimised for single precision in current release

- Each core (PPE or SPE) features a single instruction multiple data (SIMD) vector unit.

- SPEs are vector processors designed for very fast floating-point operation, at the expense of ability to run complex programs.

- Peak performance is 230 Gflops

Box 1

\section{The CUDA compatible GPU device}

The recently-introduced G80 [43] series GPU from Nvidia [44] represents the first GPU that compatible with the Nvidia Compute unified Device Architecture (CUDA)[11] platform for programming this and subsequent GPUs. Key points are:

- Highly parallel with up to 128 cores

- The G80 architecture includes texture units, which are capable of performing linear or bi-linear interpolation of arrays of floating point data.

- The current G80 device is capable only of single precision, IEEE-754 floating point arithmetic, double precision arithmetic is expected to reach the market in 2008.

- The processor also has special hardware support for reciprocal square root, exponentiation and trigonometric functions, allowing these functions to be computed with very low latency at the expense of reduced precision.

- Peak performance is 750 Gflops.

Box 2 